\title{
Photon-Mediated Interactions near a Dirac Photonic Crystal Slab
}

\author{
Erik Petrovish Navarro-Barón,* Herbert Vinck-Posada,* and Alejandro González-Tudela* \\ Cite This: ACS Photonics 2021, 8, 3209-3217 \\ Read Online
}

ABSTRACT: Dirac energy dispersions are responsible for the extraordinary transport properties of graphene. This motivated the quest for engineering such energy dispersions also in photonics, where they have been predicted to lead to many exciting phenomena. One paradigmatic example is the possibility of obtaining power-law, decoherence-free, photon-mediated interactions between quantum emitters when they interact with such photonic baths. This prediction, however, has been obtained either by using toy-model baths, which neglect polarization effects, or by restricting the emitter position to high-symmetry points of the unit cell in the case of realistic structures. Here, we develop a semianalytical theory of dipole radiation near photonic Dirac points in realistic structures that allows us to compute the effective photonmediated interactions along the whole unit cell. Using this theory, we are able to find the positions that maximize the emitter interactions and their range, finding a trade-off between them. Besides, using the polarization degree of freedom, we also find positions where the nature of the collective interactions changes from being coherent to dissipative ones. Thus, our results significantly improve the knowledge of Dirac light-matter interfaces and can serve as a guidance for future experimental designs.

KEYWORDS: photonic crystal slabs, Dirac cone dispersion, dipole-dipole interactions, quantum emitters, quantum optics, quantum simulations

\section{INTRODUCTION}

The Dirac energy spectrum of graphene is the source of many of its extraordinary electronic properties. ${ }^{1}$ This has triggered the quest to translate these energy dispersions to other systems, such as photonic crystals $(\mathrm{PhC}){ }^{2}$ by exploiting the analogy between the electronic and electromagnetic wave propagation. ${ }^{3-8}$ In this photonic context, these energy dispersions have already been predicted to lead to many nontrivial phenomena such as realizing topologically ${ }^{3}$ or pseudodiffusive ${ }^{4}$ photon transport, observing Klein tunneling, ${ }^{6}$ or enhancing the Purcell factor over large areas. ${ }^{8}$ One of the latest additions to the exciting features of Dirac photonics has been the possibility of obtaining decoherence-free, power-law interactions between emitters when many of them couple to these types of structures. ${ }^{9,10}$

Engineering power-law interactions open many exciting possibilities in the field of quantum simulation. An example is the exploration of (non-) equilibrium phenomena in frustrated spin models, ${ }^{11-17}$ where they have been predicted to lead to remarkable improvements in the transmission of quantum correlations. ${ }^{18-21}$ In free-space, photon-mediated interactions are naturally power-law (scaling with $1 / r^{3(1)}$ in the near (far) field), but they are unavoidably accompanied by dissipation. ${ }^{22,23}$ Photonic band-gaps can be used to cancel this dissipation, ${ }^{24,25}$ but at the expense of exponentially attenuating the decay of the resulting interactions. Dirac energy dispersions in two,10 and three dimensions ${ }^{26-29}$ have been recently pointed out as a way of avoiding this trade-off, showing how they lead to power-law decaying interactions without any associated dissipation thanks to the singular nature of the density of states around the Dirac points.

In the two-dimensional case, ${ }^{9,10}$ these interactions have been so far predicted to have an overall scaling with a fixed $1 / r$ decay with the distance, $r$, between emitters, restricting the models that can be explored with these quantum simulators. However, these calculations were done either using toy-model coupledresonator baths ${ }^{9}$ or fixing the emitter positions at high-symmetry points of the unit cell for the case of realistic structures, ${ }^{10}$ thus limiting the generality of the results. In this work, we go beyond these analyses and derive a general theory for realistic structures that enables us to characterize the emergent photon-mediated interactions for emitters placed at any position of the unit cell. To illustrate its power, we apply this theory to the same Dirac photonic structure analyzed in ref 10 and find several remarkable results. First, we study the positions that optimize the interaction strength beyond the one considered in ref 10 . We characterize both the regions within the dielectric and the air holes that can be used to optimize the coupling of solid-state ${ }^{30,31}$ or

Received: July 2, 2021

Published: October 21, 2021 
natural $^{32-35}$ atomic systems, respectively. Second, we find that certain positions display an effective longer-ranged decay exponent, $1 / r^{\gamma}$, with $\gamma<1$, and study the trade-off between the strength of interactions and their range. This tunability is important because it opens the exploration of other long-range interacting models beyond the ones pointed out by refs 9 and 10 or the infinite-range ones provided by standard waveguide ${ }^{36-43}$ or cavity QED setups. ${ }^{44}$ We note that such tunable power-law models have been explored in trapped ions, where they have already shown their potential, ${ }^{12}$ but restricted to one-dimensional systems. Third, by playing with the polarization degree of freedom, we also find positions where the nature of the collective interactions changes from being coherent to incoherent ones, which might lead to strong super/subradiant effects. ${ }^{45}$

The article is divided as follows: First, we introduce the formalism that connects the classical Green functions of the photonic structure with the effective quantum emitter interactions appearing in a master equation description of the emitters' dynamics. Second, we describe the Dirac-photonic structure that we will consider in this article and characterize its band structure and the properties of their eigenmodes around the Dirac point. Then, we explain the basic ingredients of the theory we develop to analyze the effective emitter interactions, which are the guided-mode expansion technique ${ }^{46}$ and the $\mathbf{k} \cdot \mathbf{p}$ method. ${ }^{47-49}$ Next, we use our theory to study the position dependence of the coupling strength, the range, and the coherent/incoherent nature of the photon-mediated interactions appearing in these systems. Finally, we will summarize our findings and point to other possible applications of our method.

\section{PHOTON-MEDIATED INTERACTIONS IN PHOTONIC CRYSTALS}

The goal of this article is to describe the emergent photonmediated interactions when several quantum emitters couple to Dirac-like light-matter interfaces, as schematically depicted in Figure 1(a). A suitable formalism to describe these interactions in these dielectric media is macroscopic QED, ${ }^{39,40,50-53}$ where the light-matter interactions are expressed in terms of the classical Green function, $\mathbf{G}\left(\mathbf{r}, \mathbf{r}^{\prime}, \omega\right)$, obtained from the following electromagnetic wave equation:

$$
\begin{aligned}
\nabla & \times \nabla \times \mathbf{G}\left(\mathbf{r}, \mathbf{r}^{\prime}, \omega\right)+\frac{\omega^{2}}{c^{2}} \varepsilon(\mathbf{r}, \omega) \mathbf{G}\left(\mathbf{r}, \mathbf{r}^{\prime}, \omega\right) \\
& =\llbracket \delta\left(\mathbf{r}-\mathbf{r}^{\prime}\right)
\end{aligned}
$$

with $\varepsilon(\mathbf{r}, \omega)$ being the permittivity of the medium. Within this formalism, and assuming that Born-Markov conditions are satisfied such that the photonic degrees of freedom can be adiabatically eliminated, ${ }^{39,40,50-53}$ the resulting emitter dynamics can be described by the following master equation:

$$
\frac{\mathrm{d} \rho(t)}{\mathrm{d} t}=-\frac{i}{\hbar}\left[H_{0}+H_{\text {eff }}, \rho\right]+\mathcal{L}_{\text {eff }}(\rho)
$$

where (i) $H_{0}$ corresponds to the independent emitters' Hamiltonian. For this article, we will assume that the emitters have a single optical transition from an optically excited state, $e$, to the ground state $g$, with frequency $\omega_{A}$ and dipole matrix element d, such that $H_{0}=\sum_{j} \hbar \omega_{A} \sigma_{e e}^{j}$, using the notation $\sigma_{\alpha \beta}^{j}=$ $|\alpha\rangle_{j}\langle\beta|$ for the emitter operators; (ii) $H_{\text {eff }}$ represents the unitary (coherent) part of the photon-mediated interactions that for two-level emitters reads

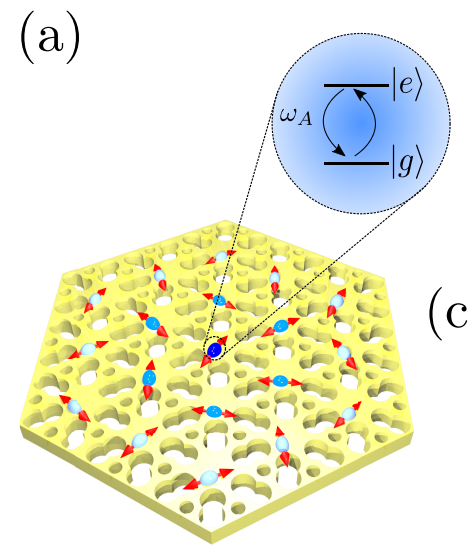

(b)

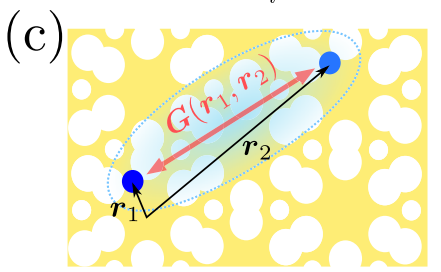

Figure 1. (a) Schematic 3D figure of the system: a collection of emitters are placed within or nearby a PhC slab with a thickness $d$. Each emitter has two levels, $|g\rangle$ and $|e\rangle$, with transition frequency $\omega_{A}$ between them. (b) Top view of the slab structure where we defined its geometric parameters: $r_{l}$ and $r_{s}$ are the radii of the inner and outer holes, $l$ is the distance between the center of the inner holes and the center of the unit cell, $a_{1}$ and $a_{2}$ are the primitive vectors for the hexagonal lattice. (c) The main focus of the work will be to obtain the two-point Green function, $\mathbf{G}\left(\mathbf{r}_{1}, \mathbf{r}_{2}\right)$, which characterizes the interaction between emitters. We will restrict it to situations where the emitters are placed at positions $\mathbf{r}_{1}$ and $\mathbf{r}_{2}=\mathbf{r}_{1}+\mathbf{R}$, where $\mathbf{R}$ is a lattice displacement vector that can be written as a linear combination of the primitive vectors $\boldsymbol{a}_{1,2}$.

$$
H_{\mathrm{eff}}=\sum_{i, j} J_{i j} \sigma_{e g}^{i} \sigma_{g e}^{j}
$$

Thus, this term yields coherent excitation exchanges between emitters at a rate $J_{i j}$, which can be harnessed, for example, to make SWAP-like gates. Finally, (iii) $\mathcal{L}_{\text {eff }}(\rho)$ describes the nonunitary (incoherent) dynamics induced by the bath that reads

$$
\mathcal{L}_{\text {eff }}(\rho)=\sum_{i j} \frac{\Gamma_{i j}}{2}\left(2 \sigma_{g e}^{i} \rho \sigma_{e g}^{j}-\sigma_{e g}^{i} \sigma_{g e}^{j} \rho-\rho \sigma_{e g}^{i} \sigma_{g e}^{j}\right)
$$

and accounts for both the individual $\left(\Gamma_{i i}\right)$ and collective $\left(\Gamma_{i \neq j}\right)$ dissipation. Despite being nonunitary, these collective dissipative terms yield strong super/subradiance effects, ${ }^{45}$ which can be harnessed to engineer decoherence-free quantum gates $^{54,55}$ or to improve multiphoton generation ${ }^{56-58}$ and absorption $^{53}$ fidelities, among other applications. Remarkably, both the coherent and incoherent terms of the master equation are related to the classical Green function as follows: ${ }^{39,40,51-53}$

$$
\begin{gathered}
J_{i j}=-\frac{\mu_{0} \omega_{A}^{2}}{\hbar} \mathbf{d}_{i}^{*} \cdot \operatorname{Re}\left[\mathbf{G}\left(\mathbf{r}_{i}, \mathbf{r}_{j}, \omega_{A}\right)\right] \cdot \mathbf{d}_{j} \\
\Gamma_{i j}=\frac{2 \mu_{0} \omega_{A}^{2}}{\hbar} \mathbf{d}_{i}^{*} \cdot \operatorname{Im}\left[\mathbf{G}\left(\mathbf{r}_{i}, \mathbf{r}_{j}, \omega_{A}\right)\right] \cdot \mathbf{d}_{j}
\end{gathered}
$$

where $\mathbf{d}_{i}$ and $\mathbf{r}_{i}$ are the optical dipole moment and position of the ith emitter, respectively. Thus, to know the photon-mediated interactions, $J_{i j}$ and $\Gamma_{i j}$ induced by a photonic media, it suffices to calculate the two-point Green function, $\mathbf{G}\left(\mathbf{r}_{i}, \mathbf{r}_{j}, \omega_{A}\right) \equiv \mathbf{G}\left(\mathbf{r}_{i}, \mathbf{r}_{j}\right)$, of the particular structure. In our case, we will only focus on the contribution of the Green function induced by the guided modes confined by the structure and neglect the contribution of the free-space modes that will mostly provide an additional individual decay channel for the regime of distances we are 
interested in. Besides, in the case of solid-state emitters, such as quantum dots, one must complement the master equation of eq 2 with additional Lindblad terms that describe pure dephasing induced by phonon modes.

In what follows, before explaining the method we develop to calculate the guided mode Green function $\mathbf{G}\left(\mathbf{r}_{i}, \mathbf{r}_{j}\right)$, let us first introduce the particular photonic structure we will use to benchmark our results.

\section{DIRAC PHOTONIC STRUCTURE}

The photonic structure that we will consider is the photonic crystal slab depicted in Figure 1, which was first introduced in ref 10. The slab consists of a hexagonal lattice of $\mathrm{GaP}\left(\varepsilon_{\mathrm{GaP}}=\right.$ $10.5625)$ with primitive vectors $a_{1 / 2}=(\sqrt{3} / 2, \pm 1 / 2) a$, where $a$ is the lattice constant, and with six inner and six outer air holes, of radii $r_{l}$ and $r_{s}$, respectively. The unit cell of the $\mathrm{PhC}$ slab is shown in detail in Figure 1(b), where $l$ denotes the distance between the center of the unit cell and the inner circles and $d$ the slab thickness. As shown in ref 10, an interesting property of this structure is that it features energetically isolated Dirac-cone dispersions at the high symmetry points $\mathbf{K}=\frac{2 \pi}{3 a}(\sqrt{3}, 1)$ and $\mathbf{K}^{\prime}=\frac{2 \pi}{3 a}(\sqrt{3},-1)$ for certain parameter regimes that are the ones we will focus on in this article.

In ref 10 the numerical analysis of the structure was done using a plane-wave expansion method. ${ }^{59}$ With the results from these numerical calculations, they proposed an empirical ansatz of the band structure $\left(\omega_{\mathbf{k}}^{(n)}\right)$ and their associated eigenmodes at the central position of the unit cell $\left(\mathbf{E}_{\mathbf{k}, n}(\mathbf{r}=0)\right)$ around the Dirac point $\mathrm{K}$. Then, using that ansatz they were able to construct the two-point Green function, $\mathbf{G}\left(\mathbf{r}_{i}, \mathbf{r}_{j}\right)$, for emitters placed at the central point of the unit cell. Here, we will use an alternative approach that consists of a combination of the guided-mode expansion (GME) technique ${ }^{46}$ and the $\mathbf{k} \cdot \mathbf{p}$ method. ${ }^{47-49}$ The former allows one to find efficient solutions for the eigenfrequencies and eigenmodes of slab geometries, whereas the later will enable us to systematically expand the energy dispersions and eigenmodes around the Dirac point and find the associated electromagnetic field at all positions of the unit cell. This will be the key advantage of our approach, since we will be able to compute the two-point Green function, and thus the emergent photon-mediated interactions, for emitters placed at any point of the unit cell.

Let us now briefly explain the main steps of our theory and apply it to characterize the photonic structure of Figure 1. The first step consists in using the GME technique. ${ }^{46}$ This method initially finds the solutions of the homogeneous slab waveguide with an effective permittivity that we denote by $\boldsymbol{h}_{\mathrm{g}, \mu}(\mathbf{r}, z)$. Using the Bloch theorem we can rewrite these solutions as $\boldsymbol{h}_{\mathrm{g}, \mu}(\mathbf{r}, z)=$ $\mathrm{e}^{i \mathbf{k} \cdot \mathbf{r}} \mathbf{U}_{\mathrm{g}, \mu}(\mathbf{r}, z)$, separating the part $\mathrm{e}^{\mathbf{i k} \cdot \mathbf{r}}$ given by the Bloch theorem from $\mathbf{U}_{\mathrm{g}, \mu}(\mathbf{r}, z)$, which provides the real space distribution within each unit cell associated with each guided mode with momentum $\mathbf{g}$ and which has the same periodicity as the original $\mathrm{PhC}$ lattice. Here, $\mathbf{g}=\mathbf{k}+\mathbf{G}$ is a combination of $\mathbf{k}$ inside the first Brillouin zone and $\mathbf{G}$ a vector of the reciprocal lattice with information about the periodicity, and $\mu$ denotes the different modes of the effective homogeneous waveguide for a given $\mathbf{g}$, which is related with the $z$-quantization of the modes. Note also that here we use $\mathbf{r}$ to express a vector in the $x y$ plane, and we will write the dependence with the $z$ coordinate when needed. Using that form for the eigenmodes of the homogeneous slab, the magnetic field of the complete photonic structure is expanded in terms of these guided modes of the homogeneous waveguide (see the Supporting Information), as follows:

$$
\mathbf{H}_{\mathbf{k}, n}(\mathbf{r}, z)=\sum_{\mathbf{G}, \mu} c_{n}(\mathbf{k}+\mathbf{G}, \mu) \boldsymbol{h}_{\mathbf{k}+\mathbf{G}, \mu}(\mathbf{r}, z)
$$

where $c_{n}(\mathbf{k}+\mathbf{G}, \mu)$ are the coefficients of expansion and $n$ denotes the different modes of the PhC slab that appear with frequency $\omega_{\mathrm{k}}^{(n)}$. Inputting this expansion into the eigenvalue problem of the Maxwell equations, one arrives at a linear matrix eigenvalue problem that can be solved numerically by truncating the size of the guided mode basis. Since we are interested in studying emitters placed at the $z=0$ plane of the slab, we can restrict our study to transverse-electric (TE)-like (even) modes, because transverse-magnetic (TM)-like ones have an electric field zero at $z=0$ and thus will not couple to the emitter.

In Figure 2(a) we plot the band structure of the TE-like modes of the structure of Figure 1 using the following geometric
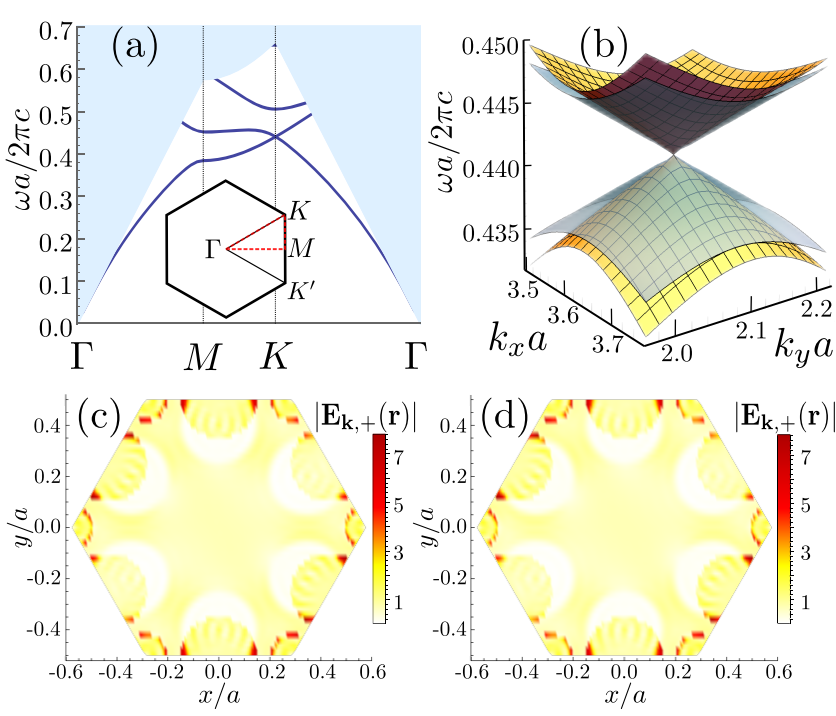

Figure 2. Band structure of the PhC slab of Figure 1. In panel (a), we show the numerical calculation of the GME method for dispersion around a particular path of the first Brillouin zone, depicted in the inset. In panel (b), we compare the $\mathbf{k} \cdot \mathbf{p}$ approximation with the GME method around the Dirac cone at $\mathbf{K}$, where the shaded blue surface corresponds to the $\mathbf{k} \cdot \mathbf{p}$ approximation and the orange surface correspond to the GME calculations. (c, d) Electric fields in the whole unit cell for an eigenmode in the upper band with momentum $\mathbf{k}=\mathbf{K}+\mathbf{q}$, with $|\mathbf{q}|=0$. $03|\mathbf{K}|$ and $\phi_{\mathrm{q}}=0$. In the two panels we compare the results obtained numerically with the GME method (c) and the fields obtained by the $\mathbf{k}$. p approximation (d). The geometric parameters for these calculations are $r_{s}=0.0833 a, r_{l}=0.15 a, l=0.4 a$, and $d=0.25 a$; we use 1165 different $\mathrm{g}$ vectors and consider three guided modes for each $\mathbf{g}$, so we have a basis with a total of 3480 guided modes.

parameters: $r_{s}=0.0833 a, r_{l}=0.15 a, l=0.4 a$, and $d=0.25 a$, calculated with the GME method using a truncation for the homogeneous waveguide basis of 3480 guided modes. There, we observe how indeed energetically isolated Dirac-cone dispersions at the K-point appear, which will be the main focus of this article. The next step consists in making a perturbative expansion around the $\mathbf{K}$-point, i.e., $\mathbf{k}=\mathbf{K}+\mathbf{q}$, for small $\mathbf{q}$, using the $\mathbf{k} \cdot \mathbf{p}$ method. The key idea of the perturbative expansion is to use the semianalytical solutions obtained from the GME method at the $\mathbf{K}$ point as the basis to express the problem at $\mathbf{k}=\mathbf{K}+\mathbf{q}$. For that, we use again the fact that the magnetic field within the $\mathrm{PhC}$ also satisfies the Bloch theorem such that we can rewrite it 
also as $\mathbf{H}_{\mathbf{k}, n}(\mathbf{r}, z)=\mathrm{e}^{i \mathbf{k} \cdot \mathbf{r}} \boldsymbol{u}_{\mathrm{k}, n}(\mathbf{r}, z)$, where $\boldsymbol{u}_{\mathbf{k}, n}(\mathbf{r}, z)$ is the Bloch periodic function, which contains the dependence of the electric field within the unit cell. Using the GME results, we can obtain the basis of the periodic function $\left\{\boldsymbol{u}_{\mathbf{K}, n}(\mathbf{r}, z)\right\}$ around $\mathbf{K}$ as follows:

$$
\boldsymbol{u}_{\mathbf{K}, n}(\mathbf{r}, z)=\sum_{\mathbf{G}, \mu} c_{n}(\mathbf{K}+\mathbf{G}, \mu) \mathbf{U}_{\mathbf{K}+\mathbf{G}, \mu}(\mathbf{r}, z)
$$

with which we can write the magnetic field for momenta around the Dirac point, $\mathbf{k}=\mathbf{K}+\mathbf{q}$, as

$$
\mathbf{H}_{\mathbf{k}, n}(\mathbf{r}, z)=\mathrm{e}^{i(\mathbf{K}+\mathbf{q}) \cdot \mathbf{r}} \sum_{j} C_{j}(\mathbf{k}, n) \boldsymbol{u}_{\mathbf{K}, j}(\mathbf{r}, z)
$$

Putting this expansion into the eigenproblem of the magnetic field, one arrives at the following equation:

$$
\sum_{j} \mathcal{H}_{l, j} C_{j}(\mathbf{k}, n)=\frac{\omega_{\mathbf{k}, n}^{2}}{c^{2}} C_{l}(\mathbf{k}, n)
$$

where the matrix elements $\mathcal{H}_{l, j}$ can have terms of order $\mathbf{q}^{0}, \mathbf{q}^{1}$, to $\mathbf{q}^{2}$, as explicitly shown in the Supporting Information. Since the Dirac points $\mathbf{K}^{(\prime)}$ have degenerate modes, we only consider the contribution of the two first bands $(l, j=1,2)$. Additionally, as we find that the energy dispersion is approximately linear, we can also neglect the order $\mathbf{q}^{2}$ terms. With these considerations the general eigenvalue problem of eq 10 reduces to a $2 \times 2$ matrix eigenproblem:

$$
\left(\begin{array}{cc}
\mathbf{q} \cdot \mathbf{P}_{11} & \mathbf{q} \cdot \mathbf{P}_{12} \\
\mathbf{q} \cdot \mathbf{P}_{12}^{*} & -\mathbf{q} \cdot \mathbf{P}_{11}
\end{array}\right)\left(\begin{array}{l}
\xi_{ \pm} \\
\eta_{ \pm}
\end{array}\right)=\Delta \lambda_{ \pm}\left(\begin{array}{l}
\xi_{ \pm} \\
\eta_{ \pm}
\end{array}\right)
$$

where $\Delta \lambda_{ \pm}=\frac{\omega_{\mathbf{k} \pm}^{2}-\omega_{\mathrm{D}}^{2}}{c^{2}}$. The subindex \pm is related to the upper and lower bands of the cone, and $\omega_{\mathrm{D}}$ corresponds to the frequency at the Dirac point, which we define numerically as the average between $\omega_{\mathbf{K}\left({ }^{\prime}\right)}^{(1)}$ and $\omega_{\mathbf{K}\left({ }^{\prime}\right)}^{(2)}$. These particular relations between the matrix elements result from the crystal symmetry called "deterministic degeneration". ${ }^{60}$ Details about $\mathbf{P}_{l, j}$ and its calculation are given in the Supporting Information.

Solving the simplified eigenvalue problem of eq 11 , we indeed obtain a linear dependence with $|\mathbf{q}|$ of the eigenvalues, as $\Delta \lambda_{ \pm}= \pm 2 \frac{v}{c} \frac{\omega_{\mathrm{D}}}{c} \mid \mathbf{q}$ l, with $v$ being the group velocity at the Dirac points, with which we can approximate the energy dispersion of the two bands as follows:

$$
\omega_{ \pm}\left(\mathbf{K}^{\left({ }^{\prime}\right)}+\mathbf{q}\right) \approx \omega_{\mathrm{D}} \pm v|\mathbf{q}|
$$

In Figure 2(b) we plot these analytical approximations (in shaded blue surface) together with the numerically obtained energy bands using the GME method (orange surface), showing a good agreement between the two. Interestingly, from the simplified eigenvalue problem obtained through the $\mathbf{k} \cdot \mathbf{p}$ approximation, we can also obtain the following magnetic and electric field expansions around the Dirac points:

$$
\begin{aligned}
& \mathbf{H}_{\mathbf{k}, \pm}(\mathbf{r}, z) \approx \mathrm{e}^{i \mathbf{q} \cdot \mathbf{r}}\left[\xi_{ \pm} \mathbf{H}_{\mathbf{k}_{0}, 1}(\mathbf{r}, z)+\eta_{ \pm} \mathbf{H}_{\mathbf{k}_{0}, 2}(\mathbf{r}, z)\right] \\
& \mathbf{E}_{\mathbf{k}, \pm}(\mathbf{r}, z) \approx \mathrm{e}^{i \mathbf{q} \cdot \mathbf{r}}\left[\xi_{ \pm} \mathbf{E}_{\mathbf{k}_{0}, 1}(\mathbf{r}, z)+\eta_{ \pm} \mathbf{E}_{\mathbf{k}_{0}, 2}(\mathbf{r}, z)\right]
\end{aligned}
$$

where $\mathbf{k}_{0}$ can be $\mathbf{K}$ or $\mathbf{K}^{\prime}$, and the subindices 1 and 2 indicate the two degenerate modes at $\mathbf{K}^{(\prime)}$. The parameters $\xi_{ \pm}$and $\eta_{ \pm}$have the following values for $\mathbf{K}^{(\prime)}$ :

$$
\begin{aligned}
& \xi_{+}=\sin \left[\frac{\phi_{\mathbf{q}}-\delta_{\mathbf{K}^{(\prime)}}}{2}\right] \\
& \eta_{+}=\mp \cos \left[\frac{\phi_{\mathbf{q}}-\delta_{\mathbf{K}^{(\prime)}}}{2}\right] \\
& \xi_{-}= \pm \cos \left[\frac{\phi_{\mathbf{q}}-\delta_{\mathbf{K}^{(\prime)}}}{2}\right] \\
& \eta_{-}=\sin \left[\frac{\phi_{\mathbf{q}}-\delta_{\mathbf{K}^{(\prime)}}}{2}\right]
\end{aligned}
$$

where the symbols \pm and $\mp$ are for $\mathbf{K} / \mathbf{K}^{\prime}$, respectively, $\tan \left(\phi_{\mathrm{q}}\right)=$ $q_{y} / q_{x}$, and the phases $\delta_{\left.\mathbf{K}^{(}\right)}= \pm \pi / 6$. In Figure 2(c,d) we plot a comparison of the electric field amplitude corresponding to a particular $\mathbf{k}$ value of the upper band along the whole unit cell obtained numerically through the GME method (c) and semianalytically using the $\mathbf{k} \cdot \mathbf{p}$ approximation (d). There, we can see how the approximation captures indeed very well the emergent physics along the whole unit cell. Finally, let us also note that despite the different appearance of the expressions of the electric field in eq 14 with respect to the empirical ansatz proposed in ref 10 for $\mathbf{r}=0$, they reproduce the same physics if we restrict our approximated fields to this position, as we explicitly show in the Supporting Information.

\section{CONSTRUCTING GREEN FUNCTIONS}

Now, we will use the analytical approximations of eqs $12-14$ to construct the Green function of the problem, as also done in ref 10. However, the advantage of our method is that the electric field expansion of eq 14 is valid for any position of the unit cell, not only for the center $\mathbf{r}=0$, and thus, we will be able to calculate $\mathbf{G}\left(\mathbf{r}_{1}, \mathbf{r}_{2}\right)$ in a more general fashion. For this work, we restrict ourselves to the situation in which the position of the two emitters differs only by a primitive lattice displacement; that is, $\mathbf{r}_{2}$ $=\mathbf{r}_{1}+\mathbf{R}$, where $\mathbf{R}=\sum_{\mathrm{i}=1}^{2} a_{i} \mathbf{a}_{i}$. Thus, we only target the calculation of $G_{\alpha \beta}\left(\mathbf{r}_{1}, \mathbf{r}_{1}+\mathbf{R}\right):=G_{\alpha \beta}\left(\mathbf{r}_{1} ; \mathbf{R}\right)$. This Green function can be calculated integrating the momentum-space Green function as follows:

$$
G_{\alpha \beta}\left(\mathbf{r}_{1} ; \mathbf{R}\right)=\iint_{\mathrm{BZ}} \frac{d^{2} \mathbf{p}}{(2 \pi)^{2}} g_{\alpha \beta}(\mathbf{p})
$$

with:

$$
g_{\alpha \beta}(\mathbf{p})=\frac{\sqrt{3} a^{2}}{2} c^{2} \sum_{n} \frac{\mathbf{E}_{\mathbf{p}, \alpha}^{(n) *}\left(\mathbf{r}_{1}\right) \mathbf{E}_{\mathbf{p}, \beta}^{(n)}\left(\mathbf{r}_{1}+\mathbf{R}\right)}{\omega_{A}^{2}-\left(\omega_{\mathbf{p}}^{(n)}\right)^{2}}
$$

where $\mathbf{E}_{\mathbf{p}, \alpha}^{(n)}\left(\mathbf{r}_{i}\right)$ is the electric-field $\alpha$-component at the $\mathbf{r}_{i}$ position associated with the eigenenergy $\omega_{\mathrm{p}}^{(n)}$ of the $n$th band. Note that due to the periodicity of the PhC (see eq 14), $\mathbf{E}_{\mathbf{p}, \alpha}^{(n) *}\left(\mathbf{r}_{1}\right) \mathbf{E}_{\mathbf{p}, \beta}^{(n)}\left(\mathbf{r}_{1}+\right.$

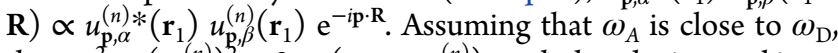
then $\omega_{A}^{2}-\left(\omega_{\mathrm{p}}^{(n)}\right)^{2} \approx 2 \omega_{A}\left(\omega_{A}-\omega_{\mathrm{p}}^{(n)}\right)$, such that the integral in eq 19 is mostly given by the contributions around the $\mathbf{K}^{(1)}$ points. This is why it is expected that the $\mathbf{k} \cdot \mathbf{p}$ expansion around the $\mathbf{K}^{(\prime)}$ points can capture well the behavior of the Green function at any point of the unit cell. Then, we can use the analytical approximations of eqs $12-14$ to obtain the Green-function components: 


$$
\begin{gathered}
G_{\alpha \beta}\left(\mathbf{r}_{1} ; \mathbf{R}\right) \approx \frac{\sqrt{3} a^{2} c^{2} \delta_{A}}{16 \omega_{A} v^{2}}\left\{i H_{0}^{(1)}\left(R \delta_{A} / v\right) \times\right. \\
{\left[\mathcal{A}_{\alpha \beta}^{\mathbf{K}}\left(\mathbf{r}_{1}\right) \mathrm{e}^{i \mathbf{K} \cdot \mathbf{R}}+\mathcal{A}_{\alpha \beta}^{\mathbf{K}^{\prime}}\left(\mathbf{r}_{1}\right) \mathrm{e}^{i \mathbf{K}^{\prime} \cdot \mathbf{R}}\right]} \\
-H_{1}^{(1)}\left(R \delta_{A} / v\right) \mathcal{B}_{\alpha \beta}^{\mathbf{K}}\left(\mathbf{r}_{1}, \phi\right) \mathrm{e}^{i \mathbf{K} \cdot \mathbf{R}} \\
\left.-H_{1}^{(1)}\left(R \delta_{A} / v\right) \mathcal{B}_{\alpha \beta}^{\mathbf{K}^{\prime}}\left(\mathbf{r}_{1}, \phi\right) \mathrm{e}^{i \mathbf{K}^{\prime} \cdot \mathbf{R}}\right\}
\end{gathered}
$$

where $\mathrm{R}=|\mathbf{R}|, \tan (\phi)=R_{y} / R_{x}, \delta_{A}=\omega_{\mathrm{D}}-\omega_{A}, H_{j}^{(1)}(r)$ are the first kind Hankel function of order $j$, and $\mathcal{A}_{\alpha \beta}^{\mathbf{K}^{(\prime)}}\left(\mathbf{r}_{1}\right), \mathcal{B}_{\alpha \beta}^{\mathbf{K}^{(1)}}\left(\mathbf{r}_{1}, \phi\right)$ are coefficients defined in terms of the direction of $\mathbf{R}$ and the electrical fields at position $\mathbf{r}_{1}$ for the two first modes of $\mathbf{K}^{(\prime)}$. More details about the explicit form of these coefficients can be found in the Supporting Information. We do not consider $G_{\alpha z}$ because it is strictly zero for emitters placed at the symmetry plane $z=0$. Finally, let us note that the Green functions associated with the circular polarized components can be reconstructed from eq 21 as follows:

$$
\begin{aligned}
& G_{\sigma_{ \pm} \sigma_{ \pm}}\left(\mathbf{r}_{1} ; \mathbf{R}\right)=\frac{G_{y y} \mp i G_{x y} \pm i G_{y x}+G_{x x}}{2} \\
& G_{\sigma_{ \pm} \sigma_{\mp}}\left(\mathbf{r}_{1} ; \mathbf{R}\right)=\frac{G_{y y} \mp i G_{x y} \mp i G_{y x}-G_{x x}}{2}
\end{aligned}
$$

In the next section, we analyze these functions in detail, putting special emphasis on the dependence of $G_{\alpha \beta}\left(\mathbf{r}_{1}, \mathbf{R}\right)$ at different places of the unit cell, since it is the main strength of our method.

\section{DIRAC-PHOTON-MEDIATED INTERACTIONS ALONG THE WHOLE UNIT CELL}

Let us start by considering the photon-mediated interactions of emitters with linearly polarized optical transitions oriented along one of the Cartesian components $(\hat{x}, \hat{y})$. As aforementioned, these interactions are given by the Green functions of eq 21, which are expressed as sums of several decaying terms scaling with different power laws. In previous works ${ }^{9,18}$ the dominant contribution was shown to be given by a $1 / r$ decay law. However, we find that when the position $\mathbf{r}_{1}$ of the emitters is varied, the interference between the different terms can give rise to even longer-ranged interactions at certain points. This is observed in Figure 3(a), where we plot $\left|G_{x x}\left(\mathbf{r}_{1} ; \mathbf{R}\right)\right|$ as a function of $R$ for a particular primitive lattice direction (other components and directions lead to qualitatively similar conclusions) for three different emitter positions in different colors, highlighted with arrows in Figure 3(b). At all emitter positions chosen, $\left|G_{x x}\left(\mathbf{r}_{1} ; \mathbf{R}\right)\right|$ have an oscillatory and power-law decay behavior. However, the decay range is faster in some positions with respect to the others. To make this more evident, we take only the maximum value of the oscillations within each period and plot them in the inset of Figure 3(a) in logarithmic scale, where one clearly observes that they follow a different power-law behavior.

To make a more detailed analysis of the change of this exponent, we make a numerical fit of the envelopes of $I G_{x x}\left(\mathbf{r}_{1}\right.$; $\mathbf{R}) \mid$ to a power law $\propto 1 /|\mathbf{R}|^{\gamma}$ at all positions of the unit cell $\mathbf{r}_{1}$ and plot the results of the fitting in Figure 3(b) in a color scale. In this figure, the white color denotes regions where the $1 / r$ behavior dominates $(\gamma=1)$, whereas red denotes scalings with longerrange decays $(\gamma<1)$. There, we observe how indeed most of the regions display the expected $1 / r$ behavior predicted in previous works. However, there also appear other regions where the
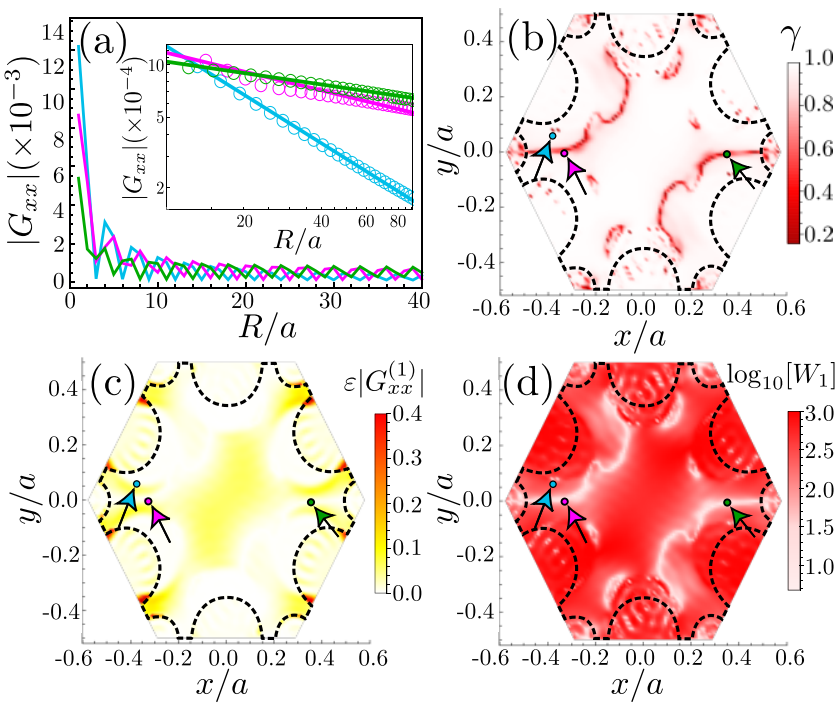

Figure 3. Behavior of $G_{x x}$ for the direction of $\phi=\pi / 6$ of a PhC structure with the same geometry parameters as in Figure 2. The transition wavelength of the emitter is fixed at $738 \mathrm{~nm}$ such that the detuning is $\delta_{A} / 2 \pi=19.5 \mathrm{GHz}$. (a) $\left|G_{x x}\right|$ as a function of $R$ for three different points in the unit cell that feature a different power-law, oscillatory, decay. The points of the unit cell chosen are depicted with arrows inside the density plots (b) $-(d)$. The inset shows a selection of the maxima of the powerlaw decay plotted in logarithmic scale (in empty circles) together with a fitting to a power law, $1 / R^{\gamma}$, showing clearly how the different points feature different decay exponent $\gamma$. (b) Behavior of the decay exponent $(\gamma)$ obtained by a numerical fitting of $\left|G_{x x}\right|$ at all positions in the unit cell. (c) Behavior of the strength of the interaction at the first neighbor position renormalized by the dielectric constant, $\varepsilon\left|G_{x x}^{(1)}\right|$, along the unit cell. (d) Ratio between the real and imaginary part of the Green function component at the first neighbor denoted by $W_{1}\left(\mathbf{r}_{1}\right)$ and plotted in a logarithmic color scale.

interference between the different terms leads to longer-ranged interactions.

Apart from the range of the interaction, another very relevant magnitude is their strength. This strength also depends on the emitter position $\mathbf{r}_{1}$, since the mode function profile also changes along the unit cell. A way of characterizing this strength is by plotting the absolute value of the photon-mediated interactions between nearest-neighboring atoms placed at different $\mathbf{r}_{1}$ positions, $\left|G_{x x}\left(\mathbf{r}, \mathbf{r}+\mathbf{a}_{1}\right)\right| \equiv\left|G_{x x}^{(1)}(\mathbf{r})\right|$. This is what we plot in Figure 3(c), multiplying it by the dielectric index $\varepsilon$ so that the strength at the air/hole regions appears on a similar color scale. From this figure, we can make two important observations: first, the optimal position to couple the emitter is not at the center of the unit cell. For emitters within the dielectric, the largest coupling strength is obtained at the regions between the air holes. For emitters lying in the holes, the coupling is maximum at regions very close to the interface. However, trapping atoms close to surfaces is generally challenging due to Casimir-Polder forces. ${ }^{25,61}$ Thus, in that case it would still be easier to place the atoms at the center of the holes, even if the coupling strength is smaller. The second important observation is that there is an apparent trade-off between the range and strength of interactions, since the red regions of panel (b) appear to be whiter (no coupling) in panel (c). We will explore this trade-off in more detail in Figure 5.

Finally, let us note that in Figure $3(a-c)$ we plot the absolute value without differentiating the contributions from the real and imaginary part of the Green function. However, as we 

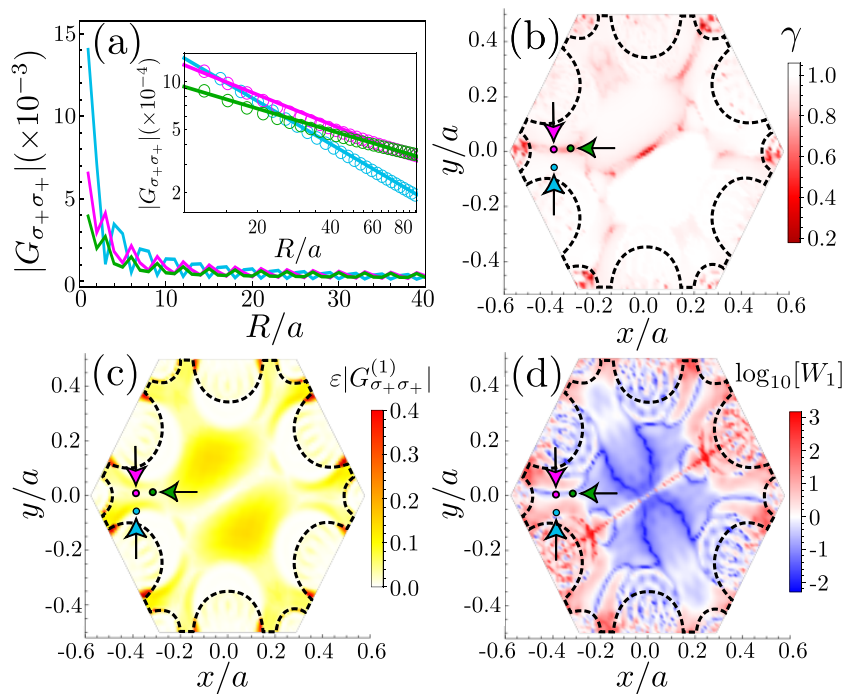

Figure 4. Behavior of $G_{\sigma_{+} \sigma_{+}}$for the direction of $\phi=\pi / 6$ for the same parameters as in Figure 3. (a) $\left|G_{\sigma_{+} \sigma_{+}}\right|$as a function of $R$ for three different points in the unit cell that feature a different power-law, oscillatory, decay. The points of the unit cell chosen are depicted with arrows inside the density plots (b) $-(d)$. The inset shows a selection of the maxima of the power-law decay plotted in logarithmic scale (in empty circles) together with a fitting to a power law, $1 / R^{\gamma}$, showing clearly how the different points feature different decay exponent $\gamma$. (b) Behavior of the decay exponent $(\gamma)$ obtained by a numerical fitting of $\left|G_{\sigma_{+} \sigma_{+}}\right|$at all positions in the unit cell. (c) Behavior of the strength of the interaction at the first neighbor position renormalized by the dielectric constant, $\varepsilon$ | $G_{\sigma_{+} \sigma_{+}}^{(1)}$ l, along the unit cell. (d) Ratio between the real and imaginary part of the Green function component at the first neighbor denoted by $W_{1}\left(\mathbf{r}_{1}\right)$ and plotted in a logarithmic color scale.
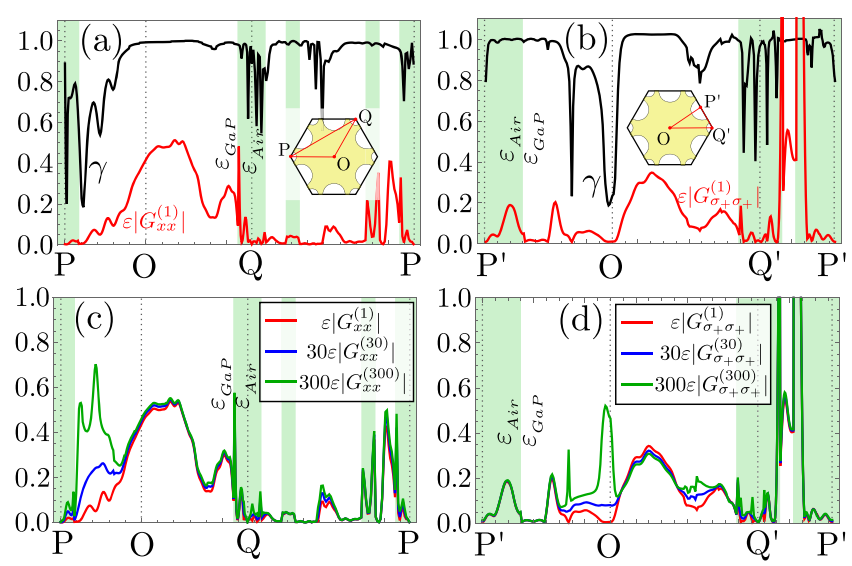

Figure 5. (a, b) The numerically obtained power-law exponent $\gamma$ and nearest-neighbor coupling strength are shown in panels $(a)$ and $(b)$ for linear $\left(\left|G_{x x}^{(1)}\right|\right)$ and circularly polarized transitions $\left(\left|G_{\sigma_{+} \sigma_{+}}^{(1)}\right|\right)$, respectively. The inset shows the path used to calculate the different curves in each polarization. (c, d) Comparison between the absolute value of the Green function at different distances for linear and circular polarization, respectively. To appropriately compare between the first, 30th, and 300th neighbor, the factor $j$ is multiplying the value $\varepsilon\left|G_{x x}^{(j)}\left(\sigma_{+} \sigma_{+}\right)\right|$Green shading represents regions along the path within the air holes.

introduced in the first part of the article, both terms give rise to very different quantum dynamics: the real part leads to purely coherent exchanges $\left(J_{i j}\right)$, whereas the imaginary part $\left(\Gamma_{i j}\right)$ yields super/subradiant effects. Thus, in Figure 3(d) we characterize which term dominates by plotting the ratio between the real and imaginary part of the nearest-neighbor position:

$$
W_{1}\left(\mathbf{r}_{1}\right)=\left|\frac{\operatorname{Re}\left[G_{x x}^{(1)}\left(\mathbf{r}_{1}\right)\right]}{\operatorname{Im}\left[G_{x x}^{(1)}\left(\mathbf{r}_{1}\right)\right]}\right|
$$

As shown in the legend accompanying the panel we use a logarithmic color scale for $W_{1}(\mathbf{r})$ where the red (blue) color denotes $W_{1}(\mathbf{r})>(<) 1$, while white denotes the transition where $W_{1}(\mathbf{r})=1$. From the figure we can clearly observe how linearly polarized emitters lead to decoherence-free photon-mediated interactions $\left(W_{1}(\mathbf{r})>1\right)$ along the unit cell. This is in accordance with the results of the literature, ${ }^{9,10}$ and it is expected because of the vanishing density of states of the photonic bath around the Dirac point, i.e., $D(E) \propto|E|$.

Let us now repeat this analysis for emitters with circularly polarized transitions. For the sake of illustration we focus again on a particular component of the Green function, i.e., $G_{\sigma_{+} \sigma_{+}}$, although the conclusions can be extrapolated to the other components. We start by plotting again $\left|G_{\sigma_{+} \sigma_{+}}\left(\mathbf{r}_{1}, \mathbf{r}_{1}+\mathbf{R}\right)\right|$ in Figure $4(\mathrm{a})$ at several representative positions $\mathbf{r}_{1}$, with different power-law scaling. This justifies again making the numerical fit to a power law $\propto 1 / \mid \mathbf{R}^{\gamma}$ at all unit cell positions, whose results we plot in Figure 4(b). There, we observe how although the dominant exponent is the expected $\gamma=1$, there are several regions with longer-ranged interactions, as it occurs for the linearly polarized transition. Then, in Figure 4(c) we plot the first-neighbor coupling strength multiplied by the permittivity of the material, i.e., $\varepsilon\left|G_{\sigma_{+} \sigma_{+}}^{1}\left(\mathbf{r}_{1}\right)\right|$. Differently from the linearly polarized case, the center of the unit cell is one of the least efficient places to couple the emitter. Again, the more favorable regions are the dielectric parts within the holes, although there also appear other places around the central part of the unit cell. At the holes, the pattern is very similar to that of linear polarizations of Figure 3(c). The main difference with respect to the linearly polarized emitters is the behavior of the real over imaginary part ratio, $W_{1}(\mathbf{r})$, defined now for $G_{\sigma_{+} \sigma_{+}}^{(1)}\left(\mathbf{r}_{1}\right)$, which we plot in Figure 4(d). There, we observe how the photonmediated interactions in most of the unit cell are dominated by the collective dissipative terms (blue regions), differently from the linearly polarized transitions (see Figure $3(d)$ ). This is a consequence of certain atomic positions of this structure satisfying $\operatorname{Re}\left[G_{x y}\right] \neq \operatorname{Re}\left[G_{y x}\right]$, which is known to lead to decay in circularly polarized transitions. ${ }^{62-65}$ This change of the coherent nature of the interactions with polarization is something that was not captured in previous analysis because they either neglected polarization effects ${ }^{9}$ or placed the emitters in positions where $G_{\sigma_{+} \sigma_{+}} \approx 0 .^{10}$

One important result from the previous analyses is the possibility of increasing the interaction range (decreasing $\gamma$ ) by placing the emitter at different positions (see panel (b) of Figures 3 and 4). However, as we also see in panels (c) of the same figures, this increase comes at the price of reducing the absolute coupling strength. Since the color scales does not let us appreciate in detail this trade-off, in Figure 5(a,b) we plot both $\gamma$ (black) and $\varepsilon\left|G_{\alpha \beta}^{(1)}(\mathbf{r})\right|$ (red) for the linear and circular polarization, respectively, and following a particular path of the unit cell depicted in the insets of this panels. There, we notice that indeed the minima of $\gamma$ coincide with positions where I $G_{\alpha \beta}^{(1)}(\mathbf{r}) \mid \approx 0$. Thus, one natural question arises: does the increase of interaction ranges compensate the decrease of coupling 
strength to couple emitters at long distances? To answer this question in Figure 5(c) and (d), we use the fit of the envelope power law for plotting a comparison of the overall coupling strength at the first (red), 30th (blue), and 300th neighbor (green), multiplying the latter by a factor so that they appear in the same scale. There, we observe in spite of this trade-off there are regions within this path where the regions with $\gamma<1$ lead to an overall better coupling at long distances than that of $\gamma=1$.

\section{CONCLUSIONS AND OUTLOOK}

Summing up, we have developed a semianalytical theory based on the guided-mode expansion and $\mathbf{k} \cdot \mathbf{p}$ method to calculate the photon-mediated interactions in Dirac light-matter interfaces. An important advantage of our theory with respect to existing ones is that it enables us to calculate such interactions when emitters are placed at all positions of the unit cell. We benchmark our theory on a particular photonic structure and find that it is possible to tune the interaction range of the emergent interactions by placing the emitter at different positions of the unit cell. Besides, we find the position that optimizes the interactions between emitters at short and long distances. Thus, we believe our theory and results can become a useful guide for future experimental designs of such Dirac lightmatter interfaces.

An interesting outlook of our theory is applying it to other nontrivial band-structure points appearing in two-dimensional PhC slabs, such as anisotropic Dirac points ${ }^{66}$ or Van Hove singularities, ${ }^{35,67-69}$ where strong super/subradiant effects ${ }^{68,69}$ or highly anisotropic coherent interactions ${ }^{70}$ have been predicted. Another research direction consists in harnessing the long-range nature of the photon mediated in such Dirac light-matter interfaces for some of the quantum information and simulation applications mentioned in the Introduction. ${ }^{11-21,71}$

\section{ASSOCIATED CONTENT}

\section{(s) Supporting Information}

The Supporting Information is available free of charge at https://pubs.acs.org/doi/10.1021/acsphotonics.1c00991.

Details on the guided-mode expansion method to calculate the eigenfrequencies and eigenmodes of the Dirac photonic structure; the $\mathbf{k} \cdot \mathbf{p}$ method to approximate the eigenfrequencies and eigenmodes around the Dirac cones; and the derivation of the Green functions by analytical integration using the expressions obtained by the $\mathbf{k} \cdot \mathbf{p}$ method; and additional results for other components of the Green function (PDF)

\section{AUTHOR INFORMATION}

\section{Corresponding Authors}

Erik Petrovish Navarro-Barón - Grupo de Superconductividad y Nanotecnología, Departamento de Física, Universidad Nacional de Colombia, Ciudad Universitaria, 26-85 Bogotá D.C., Colombia; Grupo de Óptica e Información Cuántica, Departamento de Física, , Universidad Nacional de Colombia, Ciudad Universitaria, 26-85 Bogotá D.C., Colombia; ○ orcid.org/0000-0002-9985-3841; Email: epnavarrob@ unal.edu.co

Herbert Vinck-Posada - Grupo de Superconductividad y Nanotecnología, Departamento de Física, Universidad Nacional de Colombia, Ciudad Universitaria, 26-85 Bogotá D.C., Colombia; Grupo de Óptica e Información Cuántica,
Departamento de Física, , Universidad Nacional de Colombia, Ciudad Universitaria, 26-85 Bogotá D.C., Colombia;

Email: hvinckp@unal.edu.co

Alejandro González-Tudela - Institute of Fundamental Physics IFF-CSIC, E-28006 Madrid, Spain; 이이.org/ 0000-0003-2307-6967; Email: a.gonzalez.tudela@csic.es

Complete contact information is available at:

https://pubs.acs.org/10.1021/acsphotonics.1c00991

\section{Notes}

The authors declare no competing financial interest.

\section{ACKNOWLEDGMENTS}

The authors acknowledge support from the i-COOP program from CSIC with project reference COOPA20280. A.G.T. acknowledges support from the CSIC Research Platform on Quantum Technologies PTI-001, from Spanish project PGC2018-094792-B-100 (MCIU/AEI/FEDER, EU), and from the Proyecto Sinérgico CAM 2020 Y2020/TCS-6545 (NanoQuCo-CM). E.P.N.B thanks the "Programa de becas de excelencia doctoral del bicentenario - MINCIENCIAS 2019” for financial support. H.V.P. gratefully acknowledges funding by COLCIENCIAS under the project "Impact of phonon-assisted cavity feeding process on the effective light-matter coupling in quantum electrodynamics”, HERMES 47149. A.G.T. acknowledge discussions with P. A. Huidobro about the decay of circularly polarized transitions.

\section{REFERENCES}

(1) Castro Neto, A. H.; Guinea, F.; Peres, N. M. R.; Novoselov, K. S.; Geim, A. K. The electronic properties of graphene. Rev. Mod. Phys. 2009, 81, 109-162.

(2) Joannopoulos, J. D.; Villeneuve, P. R.; Fan, S. Photonic crystals: putting a new twist on light. Nature 1997, 386, 143-149.

(3) Haldane, F. D. M.; Raghu, S. Possible realization of directional optical waveguides in photonic crystals with broken time-reversal symmetry. Phys. Rev. Lett. 2008, 100, 13904.

(4) Sepkhanov, R. A.; Bazaliy, Y. B.; Beenakker, C. W. J. Extremal transmission at the Dirac point of a photonic band structure. Phys. Rev. A: At., Mol., Opt. Phys. 2007, 75, 63813.

(5) Zandbergen, S. R.; de Dood, M. J. A. Experimental Observation of Strong Edge Effects on the Pseudodiffusive Transport of Light in Photonic Graphene. Phys. Rev. Lett. 2010, 104, 43903.

(6) Bahat-Treidel, O.; Peleg, O.; Grobman, M.; Shapira, N.; Segev, M.; Pereg-Barnea, T. Klein tunneling in deformed honeycomb lattices. Phys. Rev. Lett. 2010, 104, 063901.

(7) Zhang, X. Observing Zitterbewegung for Photons near the Dirac Point of a Two-Dimensional Photonic Crystal. Phys. Rev. Lett. 2008, $100,113903$.

(8) Bravo-Abad, J.; Joannopoulos, J. D.; Soljačić, M. Enabling singlemode behavior over large areas with photonic Dirac cones. Proc. Natl. Acad. Sci. U. S. A. 2012, 109, 9761-9765.

(9) González-Tudela, A.; Cirac, J. Exotic quantum dynamics and purely long-range coherent interactions in Dirac conelike baths. Phys. Rev. A: At., Mol., Opt. Phys. 2018, 97, DOI: 10.1103/PhysRevA.97.043831

(10) Perczel, J.; Lukin, M. D. Theory of dipole radiation near a Dirac photonic crystal. Phys. Rev. A: At., Mol., Opt. Phys. 2020, 101, 033822.

(11) Hauke, P.; Tagliacozzo, L. Spread of Correlations in Long-Range Interacting Quantum Systems. Phys. Rev. Lett. 2013, 111, 207202.

(12) Richerme, P.; Gong, Z.-X.; Lee, A.; Senko, C.; Smith, J.; FossFeig, M.; Michalakis, S.; Gorshkov, A. V.; Monroe, C. Non-local propagation of correlations in quantum systems with long-range interactions. Nature 2014, 511, 198-201. 
(13) Gong, Z.-X.; Maghrebi, M. F.; Hu, A.; Foss-Feig, M.; Richerme, P.; Monroe, C.; Gorshkov, A. V. Kaleidoscope of quantum phases in a long-range interacting spin-1 chain. Phys. Rev. B: Condens. Matter Mater. Phys. 2016, 93, 205115.

(14) Maghrebi, M. F.; Gong, Z.-X.; Foss-Feig, M.; Gorshkov, A. V. Causality and quantum criticality in long-range lattice models. Phys. Rev. B: Condens. Matter Mater. Phys. 2016, 93, 125128.

(15) Koffel, T.; Lewenstein, M.; Tagliacozzo, L. Entanglement Entropy for the Long-Range Ising Chain in a Transverse Field. Phys. Rev. Lett. 2012, 109, 267203.

(16) Vodola, D.; Lepori, L.; Ercolessi, E.; Gorshkov, A. V.; Pupillo, G. Kitaev Chains with Long-Range Pairing. Phys. Rev. Lett. 2014, 113, 156402.

(17) Kastner, M. Diverging Equilibration Times in Long-Range Quantum Spin Models. Phys. Rev. Lett. 2011, 106, 130601.

(18) Eldredge, Z.; Gong, Z.-X.; Young, J. T.; Moosavian, A. H.; FossFeig, M.; Gorshkov, A. V. Fast Quantum State Transfer and Entanglement Renormalization Using Long-Range Interactions. Phys. Rev. Lett. 2017, 119, 170503.

(19) Kuwahara, T.; Saito, K. Strictly Linear Light Cones in LongRange Interacting Systems of Arbitrary Dimensions. Phys. Rev. X 2020, 10, 031010 .

(20) Tran, M. C.; Deshpande, A.; Guo, A. Y.; Lucas, A.; Gorshkov, A. V. Optimal State Transfer and Entanglement Generation in Power-law Interacting Systems. arXiv:2010.02930, 2010.

(21) Tran, M. C.; Guo, A. Y.; Baldwin, C. L.; Ehrenberg, A.; Gorshkov, A. V.; Lucas, A. Lieb-Robinson light cone for power-law interactions. Phys. Rev. Lett. 2021, DOI: 10.1103/PhysRevLett.127.160401.

(22) Lehmberg, R. H. Radiation from an \$N\$-Atom System. I. General Formalism. Phys. Rev. A: At., Mol., Opt. Phys. 1970, 2, 883-888. (23) Lehmberg, R. H. Radiation from an \$N\$-Atom System. II. Spontaneous Emission from a Pair of Atoms. Phys. Rev. A: At., Mol., Opt. Phys. 1970, 2, 889-896.

(24) Douglas, J. S.; Habibian, H.; Hung, C.-L.; Gorshkov, A. V.; Kimble, H. J.; Chang, D. E. Quantum many-body models with cold atoms coupled to photonic crystals. Nat. Photonics 2015, 9, 326-331.

(25) González-Tudela, A.; Hung, C.-L.; Chang, D.; Cirac, J.; Kimble, $\mathrm{H}$. Subwavelength vacuum lattices and atom-atom interactions in twodimensional photonic crystals. Nat. Photonics 2015, 9, 320.

(26) González-Tudela, A.; Cirac, J. Non-Markovian quantum optics with three-dimensional state-dependent optical lattices. Quantum 2018, 2,97.

(27) Ying, L.; Zhou, M.; Mattei, M.; Liu, B.; Campagnola, P.; Goldsmith, R. H.; Yu, Z. Extended range of dipole-dipole interactions in periodically structured photonic media. Phys. Rev. Lett. 2019, 123, 173901.

(28) García-Elcano, I.; González-Tudela, A.; Bravo-Abad, J. Tunable and Robust Long-Range Coherent Interactions between Quantum Emitters Mediated by Weyl Bound States. Phys. Rev. Lett. 2020, 125, 163602.

(29) García-Elcano, I.; Bravo-Abad, J.; González-Tudela, A. Lightmatter interactions near photonic Weyl points. Phys. Rev. A: At., Mol., Opt. Phys. 2021, 103, 033511.

(30) Evans, R. E.; Bhaskar, M. K.; Sukachev, D. D.; Nguyen, C. T.; Sipahigil, A.; Burek, M. J.; Machielse, B.; Zhang, G. H.; Zibrov, A. S.; Bielejec, E. Photon-mediated interactions between quantum emitters in a diamond nanocavity. Science 2018, 362, 662-665.

(31) Lodahl, P.; Mahmoodian, S.; Stobbe, S. Interfacing single photons and single quantum dots with photonic nanostructures. Rev. Mod. Phys. 2015, 87, 347-400.

(32) Goban, A.; Hung, C.-L.; Yu, S.-P.; Hood, J. D.; Muniz, J. A.; Lee, J. H.; Martin, M. J.; McClung, A. C.; Choi, K. S.; Chang, D. E.; Painter, O.; Kimblemblrm, H. J. Atom-Light Interactions in Photonic Crystals. Nat. Commun. 2014, 5, 3808.

(33) Thompson, J. D.; Tiecke, T. G.; de Leon, N. P.; Feist, J.; Akimov, A. V.; Gullans, M.; Zibrov, A. S.; Vuletic, V.; Lukin, M. D. Coupling a Single Trapped Atom to a Nanoscale Optical Cavity. Science 2013, 340, $1202-1205$.
(34) Kim, M. E.; Chang, T. H.; Fields, B. M.; Chen, C. A.; Hung, C. L. Trapping single atoms on a nanophotonic circuit with configurable tweezer lattices. Nat. Commun. 2019, DOI: 10.1038/s41467-01909635-7.

(35) Yu, S. P.; Muniz, J. A.; Hung, C. L.; Kimble, H. J. Twodimensional photonic crystals for engineering atom-light interactions. Proc. Natl. Acad. Sci. U. S. A. 2019, 116, 12743-12751.

(36) le Kien, F.; Dutta Gupta, S.; Balykin, V. I.; Hakuta, K. Spontaneous emission of a cesium atom near a nanofiber: Efficient coupling of light to guided modes. Phys. Rev. A: At., Mol., Opt. Phys. 2005, 72, 32509.

(37) le Kien, F.; Rauschenbeutel, A. Propagation of nanofiber-guided light through an array of atoms. Phys. Rev. A: At., Mol., Opt. Phys. 2014, 90,063816 .

(38) Lalumiére, K.; Sanders, B. C.; van Loo, A. F.; Fedorov, A.; Wallraff, A.; Blais, A. Input-output theory for waveguide QED with an ensemble of inhomogeneous atoms. Phys. Rev. A: At., Mol., Opt. Phys. 2013, 88, 043806.

(39) Gonzalez-Tudela, A.; Martin-Cano, D.; Moreno, E.; MartinMoreno, L.; Tejedor, C.; Garcia-Vidal, F. J. Entanglement of two qubits mediated by one-dimensional plasmonic waveguides. Phys. Rev. Lett. 2011, 106, DOI: 10.1103/PhysRevLett.106.020501.

(40) Dzsotjan, D.; Sørensen, A. S.; Fleischhauer, M. Quantum emitters coupled to surface plasmons of a nanowire: A Green's function approach. Phys. Rev. B: Condens. Matter Mater. Phys. 2010, 82, 75427.

(41) Chang, D. E.; Jiang, L.; Gorshkov, A. V.; Kimble, H. J. Cavity QED with atomic mirrors. New J. Phys. 2012, 14, 63003.

(42) Lodahl, P.; Mahmoodian, S.; Stobbe, S.; Rauschenbeutel, A.; Schneeweiss, P.; Volz, J.; Pichler, H.; Zoller, P. Chiral quantum optics. Nature 2017, 541, 473.

(43) Arranz Regidor, S.; Crowder, G.; Carmichael, H.; Hughes, S. Modeling quantum light-matter interactions in waveguide QED with retardation, nonlinear interactions, and a time-delayed feedback: Matrix product states versus a space-discretized waveguide model. Phys. Rev. Res. 2021, 3, DOI: 10.1103/PhysRevResearch.3.023030.

(44) Ritsch, H.; Domokos, P.; Brennecke, F.; Esslinger, T. Cold atoms in cavity-generated dynamical optical potentials. Rev. Mod. Phys. 2013, $85,553-601$.

(45) Dicke, R. H. Coherence in Spontaneous Radiation Processes. Phys. Rev. 1954, 93, 99.

(46) Andreani, L. C.; Gerace, D. Photonic-crystal slabs with a triangular lattice of triangular holes investigated using a guided-mode expansion method. Phys. Rev. B: Condens. Matter Mater. Phys. 2006, 73, 235114.

(47) Lee, W. M. Theory of Photonic Band Gap Materials. MPhil thesis, Chinese University of Hong Kong, 1994.

(48) Hui, P. M.; Lee, W. M.; Johnson, N. F. Theory of scalar wave propagation in periodic composites: A k.p approach. Solid State Commun. 1994, 91, 65-69.

(49) Sipe, J. E. Vector k.p approach for photonic band structures. Phys. Rev. E: Stat. Phys., Plasmas, Fluids, Relat. Interdiscip. Top. 2000, 62, $5672-5677$.

(50) Gruner, T.; Welsch, D. G. Green-function approach to the radiation-field quantization for homogeneous and inhomogeneous Kramers-Kronig dielectrics. Phys. Rev. A: At., Mol., Opt. Phys. 1996, 53, $1818-1829$.

(51) Dung, H. T.; Knöll, L.; Welsch, D.-G. Resonant dipole-dipole interaction in the presence of dispersing and absorbing surroundings. Phys. Rev. A: At., Mol., Opt. Phys. 2002, 66, 63810.

(52) Buhmann, S. Y.; Welsch, D.-G. Dispersion forces in macroscopic quantum electrodynamics. Prog. Quantum Electron. 2007, 31, 51-130.

(53) Asenjo-Garcia, A.; Hood, J. D.; Chang, D. E.; Kimble, H. J. Atomlight interactions in quasi-one-dimensional nanostructures: A Green'sfunction perspective. Phys. Rev. A: At., Mol., Opt. Phys. 2017, 95, 33818.

(54) Paulisch, V.; Kimble, H.; González-Tudela, A. Universal quantum computation in waveguide QED using decoherence free subspaces. New J. Phys.. 2016, 18, 043041. 
(55) Kockum, A. F.; Johansson, G.; Nori, F. Decoherence-Free Interaction between Giant Atoms in Waveguide Quantum Electrodynamics. Phys. Rev. Lett. 2018, 120, 140404.

(56) González-Tudela, A.; Paulisch, V.; Chang, D.; Kimble, H.; Cirac, J. Deterministic Generation of Arbitrary Photonic States Assisted by Dissipation. Phys. Rev. Lett. 2015, 115, DOI: 10.1103/PhysRevLett.115.163603.

(57) González-Tudela, A.; Paulisch, V.; Kimble, H.; Cirac, J. Efficient Multiphoton Generation in Waveguide Quantum Electrodynamics. Phys. Rev. Lett. 2017, 118, DOI: 10.1103/PhysRevLett.118.213601.

(58) Paulisch, V.; Perarnau-Llobet, M.; González-Tudela, A., Cirac, J. Quantum metrology with one-dimensional superradiant photonic states. Phys. Rev. A: At., Mol., Opt. Phys. 2019, 99, DOI: 10.1103/ PhysRevA.99.043807.

(59) Johnson, S. G.; Joannopoulos, J. D. Block-iterative frequencydomain methods for Maxwell's equations in a planewave basis. Opt. Express 2001, 8, 173-190.

(60) Mei, J.; Wu, Y.; Chan, C. T.; Zhang, Z. Q. First-principles study of Dirac and Dirac-like cones in phononic and photonic crystals. Phys. Rev. B: Condens. Matter Mater. Phys. 2012, 86, 1-7.

(61) Hung, C.-L.; Meenehan, S. M.; Chang, D. E.; Painter, O.; Kimble, H. J. Trapped atoms in one-dimensional photonic crystals. New J. Phys. 2013, 15, 83026.

(62) Tang, Y.; Cohen, A. E. Optical chirality and its interaction with matter. Phys. Rev. Lett. 2010, 104, 163901.

(63) García-Etxarri, A.; Dionne, J. A. Surface-enhanced circular dichroism spectroscopy mediated by nonchiral nanoantennas. Phys. Rev. B: Condens. Matter Mater. Phys. 2013, 87, 235409.

(64) Yoo, S.; Park, Q. H. Chiral Light-Matter Interaction in Optical Resonators. Phys. Rev. Lett. 2015, 114, 203003.

(65) Neuman, T.; Wang, D. S.; Narang, P. Nanomagnonic Cavities for Strong Spin-Magnon Coupling and Magnon-Mediated Spin-Spin Interactions. Phys. Rev. Lett. 2020, 125, 247702.

(66) Redondo-Yuste, J.; de Paz, M. B.; Huidobro, P. A.; GonzálezTudela, A. Quantum electrodynamics in anisotropic and tilted Dirac photonic lattices. arXiv, 2021.

(67) Galve, F.; Mandarino, A.; Paris, M. G. A.; Benedetti, C.; Zambrini, R. Microscopic description for the emergence of collective dissipation in extended quantum systems. Sci. Rep. 2017, 7, DOI: $10.1038 /$ srep42050.

(68) González-Tudela, A.; Cirac, J. Quantum Emitters in TwoDimensional Structured Reservoirs in the Nonperturbative Regime. Phys. Rev. Lett. 2017, 119, DOI: 10.1103/PhysRevLett.119.143602.

(69) González-Tudela, A.; Cirac, J. Markovian and non-Markovian dynamics of quantum emitters coupled to two-dimensional structured reservoirs. Phys. Rev. A: At., Mol., Opt. Phys.. 2017, 96, DOI: 10.1103/ PhysRevA.96.043811.

(70) González-Tudela, A.; Galve, F. Anisotropic quantum emitter interactions in two-dimensional photonic-crystal baths. ACS Photonics 2019, 6, 221-229.

(71) Shahmoon, E.; Kurizki, G. Nonradiative interaction and entanglement between distant atoms. Phys. Rev. A: At., Mol., Opt. Phys. 2013, 87, 33831. 